\title{
Aip regulates CAMP signalling and GH secretion in GH3 cells
}

\author{
R Formosa', A Xuereb-Anastasi ${ }^{2}$ and J Vassallo ${ }^{1,3}$ \\ ${ }^{1}$ Department of Medicine, Faculty of Medicine and Surgery, Mater Dei Hospital, University of Malta, Block A, \\ Level 0, Msida MSD2080, Malta \\ ${ }^{2}$ Department of Applied Biomedical Science, Faculty of Health Sciences, University of Malta, Msida, Malta \\ ${ }^{3}$ Neuroendocrine Clinic, Department of Medicine, Mater Dei Hospital, Msida, Malta
}

Correspondence

should be addressed

to J Vassallo

Email

josanne.vassallo@um.edu.mt

\begin{abstract}
Mutations in the aryl hydrocarbon receptor-interacting protein (AIP) gene have been linked to predisposition to pituitary adenomas. However, the mechanism by which this occurs remains unknown. AIP interacts with a number of interesting proteins, including members of the CAMP signalling pathway that has been shown to be consistently altered in pituitary tumours. The functional role of Aip was investigated using both over-expression and knock down of Aip in GH3 cells. CAMP signalling and its downstream effectors, including GH secretion, were then investigated. CAMP signalling was analysed using CAMP assays, CAMP-response element-promoter luciferase reporter assays, real-time PCR and finally secreted GH quantification. Over-expression of wild-type (WT)-Aip reduced forskolin-induced cAMP signalling at the total CAMP level, luciferase reporter activity and target gene expression, when compared with empty vector and the non-functional R304X mutant. Additionally, GH secretion was reduced in WT-Aip over-expressing GH3 cells treated with forskolin. Knock down of endogenous Aip resulted in increased cAMP signalling but a decrease in GH secretion was also noted. Inhibition of phosphodiesterase activity using general and selective inhibitors did not completely ablate the effect of Aip on forskolin-augmented CAMP signalling. A mechanism by which Aip acts as a tumour suppressor, by maintaining a low CAMP signalling and concentration, is suggested. Mutations of Aip render the protein incapable of such activity. This effect appears not to be mediated by the AIP-PDE interaction, suggesting the involvement of other interacting partners in mediating this outcome.
\end{abstract} Key Words

- AIP

- function

- CAMP

- pituitary tumours

\section{Introduction}

Pituitary tumours represent the most common neoplasms of the brain. Screening of familial cases of pituitary tumours identified germ-line mutations in the aryl hydrocarbon receptor-interacting protein (Aip), which occur almost exclusively in somatotroph and lactotroph adenomas (Vierimaa et al. 2006). A recent multi-centre study revealed that patients with Aip mutation have earlier onset, characteristically more aggressive tumours that do not respond well to treatment and require more surgical interventions (Daly et al. 2010). Most common gene alterations result in amino acid substitutions or a truncated AIP protein particularly within the C-terminal, which contains three tetratricopeptide repeats (TRP) responsible for protein-protein interactions (Leontiou et al. 2008). The AIP is a co-chaperone protein that binds to a number of interacting partners, including the phosphodiesterases PDE4A5 and PDE2A, the inhibitor of apoptosis survivin, several nuclear receptors and G-proteins $\alpha$-subunit $13\left(\mathrm{G} \alpha_{13}\right)$ and $\alpha$-subunit q $\left(\mathrm{G} \alpha_{\mathrm{q}}\right)$ (Bolger et al. 2003, Kang \& Altieri 2006, de Oliviera et al. 
2007, Beischlag et al. 2008, Nakata et al. 2009). The ability to repress proliferation in cell lines, the frequent loss of heterozygozity and inactivation of AIP protein in pituitary adenomas suggest a role as a tumour suppressor (Leontiou et al. 2008). Although it is likely that AIP functions through one or more of its interacting partners in bringing about the anti-proliferative behaviour, the precise mechanism/s remains unknown.

The interaction between AIP, phosphodiesterases and G-proteins led us to focus on the cAMP signalling pathway, which is frequently de-regulated in pituitary adenomas. Mutations in the GNAS and PKRAR1A genes, associated with McCune-Albright syndrome and Carney complex respectively, lead to increased cAMP signalling (Horvath \& Stratakis 2008). Alteration of cAMP levels has been proven to be a trademark of endocrine neoplasms, particularly in secretory tumours (Suzuki et al. 1999, Lania et al. 2001, 2004, Pertuit et al. 2009), probably due to the role of this signalling pathway in hormone production and release and in regulating cellular physiology and proliferation (Ramirez et al. 1999). G-proteins and phosphodiesterases play an important role in regulating cAMP signalling through the respective production and degradation of cAMP. Given the interaction between AIP and several members of the cAMP signalling pathway, we decided to focus our study on the interaction between AIP and this mitogenic signalling pathway.

In an attempt to identify mechanisms by which AIP suppresses tumour growth, several studies were undertaken, including the role of survivin in directing cell apoptosis. Real-time PCR and immunohistochemistry revealed that survivin expression is extremely low in local pituitary tumours, suggesting that its role and, therefore, the significance of its interaction with AIP, is minimal (Formosa et al. 2012). In this study, the effect of over-expressing and knocking down AIP proteins directly on cAMP signalling and GH secretion in the GH3 cell line was analysed. We provide evidence that AIP limits cAMP signalling and this in turn effects GH secretion from GH3 cells.

\section{Materials and methods}

\section{Reagents, plasmids, siRNAs, cell lines and antibodies}

Forskolin and rolipram were purchased from Ascent Scientific (Bristol, UK) while 3-isobutyl-1-methylxanthine (IBMX) was purchased from Sigma. pcDNA3-WT-AIP-myc and pcDNA3-R304X-AIP-myc plasmids were generously donated by Prof. Marta Korbonits. The Rip1-Cre-Luc plasmid (Chepurny \& Holz 2007) was generously donated by Dr George Holz (Upstate Medical University, New York). GH3 cells were also donated by Prof. Korbonits and Hela cells were donated by Dr Anthony Fenech and both maintained in DMEM with 10\% serum and $1 \%$ penicillin/streptomycin. XAP2 (AIP) and $\beta$-actin antibodies were obtained from Novus Biologicals (Littleton, CO, USA). siRNA specific to rat Aip and non-targeting siRNA were bought from Dharmacon (ThermoFisher Scientific, Pittsburgh, PA, USA).

\section{Transfections and western blots}

GH3 cells were transfected with either wild-type (WT)-AIP, pcDNA3 empty vector (EV), R304X mutant AIP plasmids or rat Aip siRNA or non-targeting siRNA. Plasmids were transfected using Fugene (Promega) while siRNAs were transfected using Lipofectamine RNAiMAX (Life Technologies). Details regarding transfection conditions can be found (data not shown). Western blots were carried out using a standard protocol (data not shown). Normalisation of transfection efficiency was carried out by real-time PCR and western blot of AIP mRNA and protein $48 \mathrm{~h}$ post-transfection.

\section{Luciferase activity assays}

GH3 and Hela cells were plated in 24-well plates and transfection of plasmids and/or siRNAs was carried out as described, using $0.5 \mu \mathrm{g}$ AIP plasmid, $0.5 \mu \mathrm{g}$ Rip1-Cre-Luc plasmid and $0.0125 \mu \mathrm{g}$ Renilla plasmid per well with five replicates per treatment. Transfection of $30 \mathrm{pmol}$ siRNA per well was carried out $8 \mathrm{~h}$ before transfection of plasmids in GH3 cells only. After $40 \mathrm{~h}$, a quarter of the wells were left untreated, a quarter were treated with $1 \mu \mathrm{M}$ forskolin only, a further quarter were treated with $10 \mu \mathrm{M}$ IBMX, followed by $1 \mu \mathrm{M}$ forskolin after $30 \mathrm{~min}$ while the last quarter of the wells were treated with $10 \mu \mathrm{M}$ rolipram, followed by $1 \mu \mathrm{M}$ forskolin after $30 \mathrm{~min}$. After $8 \mathrm{~h}$, the medium was removed, cells were washed with cold PBS and $100 \mu \mathrm{l}$ passive lysis buffer were added to each well. The Dual Luciferase Assay kit (Promega) was used to measure luciferase activity, read by luminometer (TD-20/20, Turner Biosystems, Promega, Madison, WI, USA).

\section{RNA extraction, RT and real-time PCR}

GH3 cells were seeded in 24-well plates and transfection with all four plasmids and/or siRNA was carried out after $24 \mathrm{~h}$, followed by forskolin or IBMX/rolipram, and forskolin treatment after $48 \mathrm{~h}$ was carried out as previously

Published by Bioscientifica Ltd. 
described earlier. Three hours after the addition of forskolin, the cells were lysed in RLT buffer and RNA was extracted using the RNeasy Mini Kit (Qiagen). RT was carried out with the GoScript kit (Promega) and real-time PCR was carried out for the rat inhibitor of DNA binding-1 (Id1) gene using ribonuclease inhibitor 1 ( $R i)$ gene as a housekeeping gene in the ABI7300 real-time thermocycler (Applied Biosystems). Quantitative PCR for endogenous and transfected AIP was also carried out.

\section{Total intracellular CAMP production and secreted GH quantification}

GH3 cells were seeded in 24-well plates and transfected after $24 \mathrm{~h}$ in serum-free medium. After $48 \mathrm{~h}$, cells were treated with $1 \mu \mathrm{M}$ forskolin only, $10 \mu \mathrm{M}$, or rolipram followed by $1 \mu \mathrm{M}$ forskolin after $30 \mathrm{~min}$ or left untreated. After $1 \mathrm{~h}$, the medium was collected and stored for GH quantification. The cells were washed with PBS and lysed. Endogenous cAMP was quantified using the Parameter Cyclic AMP kit (KGE002B, R\&D Systems, Minneapolis, $\mathrm{MN}$, USA) following the manufacturer's instructions. Secreted GH in the medium was measured using Rat Growth Hormone ELISA Assay (KRC5311, Invitrogen). Cell counting was carried out using standard haemocytometer readings.

\section{Statistical analysis}

All data were analysed using SPSS Statistics version 17 (IBM). Kolmogorov-Smirnov test was carried out on all the raw data to determine the normal distribution of the data. All data were proven to be parametric and therefore oneway ANOVA was used for the proliferation assays, the luciferase reporter assays, real-time PCR, cAMP and GH quantification. Statistical significance was set at the $5 \%$ confidence level.

\section{Results}

The scope of this study was to investigate the influence of AIP on the overall cAMP status of the cell and its downstream effectors using both over-expression of WTand mutant Aip and knock down of endogenous AIP to analyse whether any observed activity would be reversed upon knock out of the Aip expression. In order to do so, three methods were used. First, a cAMP-response element (CRE)-driven luciferase assay using the rat insulin gene 1 promoter (Rip1) was used. Next, to determine that this effect was not due to the transient transfection of vectors only, the expression of a known cAMP-responsive gene, the Id1 gene was analysed. Id1 was selected as a representative of cAMP target genes due to the wellcharacterised CRE in its promoter region and the fact that

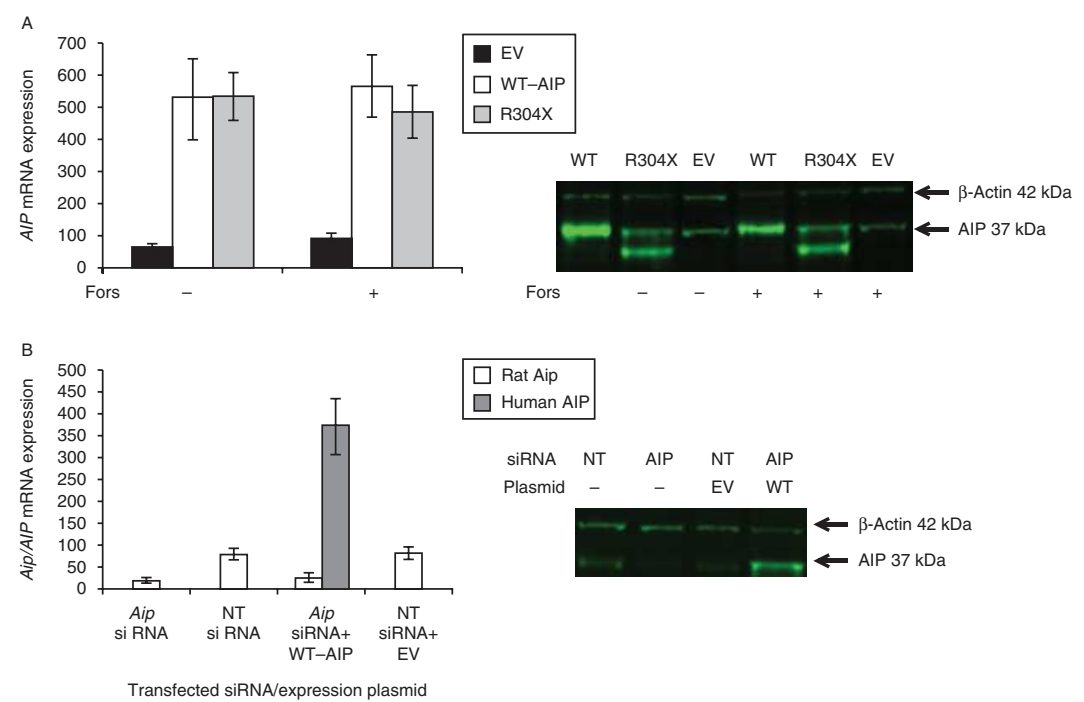

\section{Figure 1}

Real-time PCR and western blot analysis of Aip over-expression (A) and endogenous Aip knock down (B). Endogenous Aip expression was not altered by the addition of $1 \mu \mathrm{M}$ forskolin. $\mathrm{GH} 3$ cells were transfected with empty vector (EV), wild-type (WT)-AIP or R304X mutant Aip expression plasmids and lysed after $48 \mathrm{~h}$. In forskolin-treated cells, forskolin was added $6 \mathrm{~h}$ before lysis and RNA/protein extraction (A). In Aip knock down, GH3 cells were transfected with Aip or non-targeting (NT) siRNA and lysed after $48 \mathrm{~h}$. GH3 cells were also co-transfected with either Aip siRNA and WT-AIP or NT siRNA and EV as controls. Bars represent means of three separate experiments with three replicates per experiment real-time PCR, which were normalised against $R i$ rat gene. Error bars indicate S.E.M. (Fors, forskolin). Full colour version of this figure available via http://dx.doi.org/10.1530/ERC-13-0043.

Published by Bioscientifica Ltd. 
a number of studies have similarly used Id1 expression as an indication of CREB transcription factor activity (Zhang et al. 2005, Ohta et al. 2008, San-Marina et al. 2008, Yang et al. 2010). Finally, total cell cAMP was also quantified using a standard validated kit. In addition, forskolin, an adenylate cyclase activator, was used to stimulate cAMP production and therefore to function as a positive control, while IBMX, a general phosphodiesterases inhibitor, was used to verify whether any effect of the over-expressed AIP protein was being mediated by either PDE4A5 or PDE2A proteins. Rolipram, a PDE4-specific inhibitor was also used to selectively impede the function of PDE4A5.

\section{Activation of CAMP by $1 \mu \mathrm{M}$ forskolin does not induce AIP expression in GH3 cells}

Treatment with $1 \mu \mathrm{M}$ forskolin did not increase the endogenous Aip expression significantly in GH3 cells, while transient transfection increased vector AIP mRNA and protein by $\sim 50$-fold (Fig. $1 \mathrm{~A}$ and B). Forskolin did not have any effect on either the endogenous AIP expression or the pcDNA3 vector-driven AIP expression. The success of Aip knock down using a four oligonucleotide primer set can also be seen in the western blot and real-time analysis (Fig. 1C and D). As observed by relative quantification using real-time PCR, $\sim 70-80 \%$ of the endogenous AIP expression was knocked down successfully in these experiments.

\section{WT-AIP over-expression reduces forskolin-induced CAMP-driven transcription}

Luciferase assays carried out in GH3 and Hela cells after both over-expression of WT and mutant Aip and knock down with rat Aip siRNA in GH3 cells can be seen in Fig. 2 . Over-expression of WT-Aip did not alter basal levels of luciferase activity significantly compared with the EV or mutant Aip in both rat and human cell lines. However, WT-AIP protein over-expression was able to reduce forskolin-induced CRE-driven luciferase transcription/ activity significantly when compared with $\mathrm{EV}$ and the R304X-truncated AIP in GH3 cells and Hela cells equally (Fig. 2A and B). Interestingly, this behaviour is mostly retained when cells are pre-treated with IBMX, indicating that inhibition of phosphodiesterases does not influence the ability of WT-Aip to decrease CRE-driven transcription of the luciferase gene. Rolipram, a selective PDE4 inhibitor, was also unable to reverse the inhibitory activity or WT-Aip.
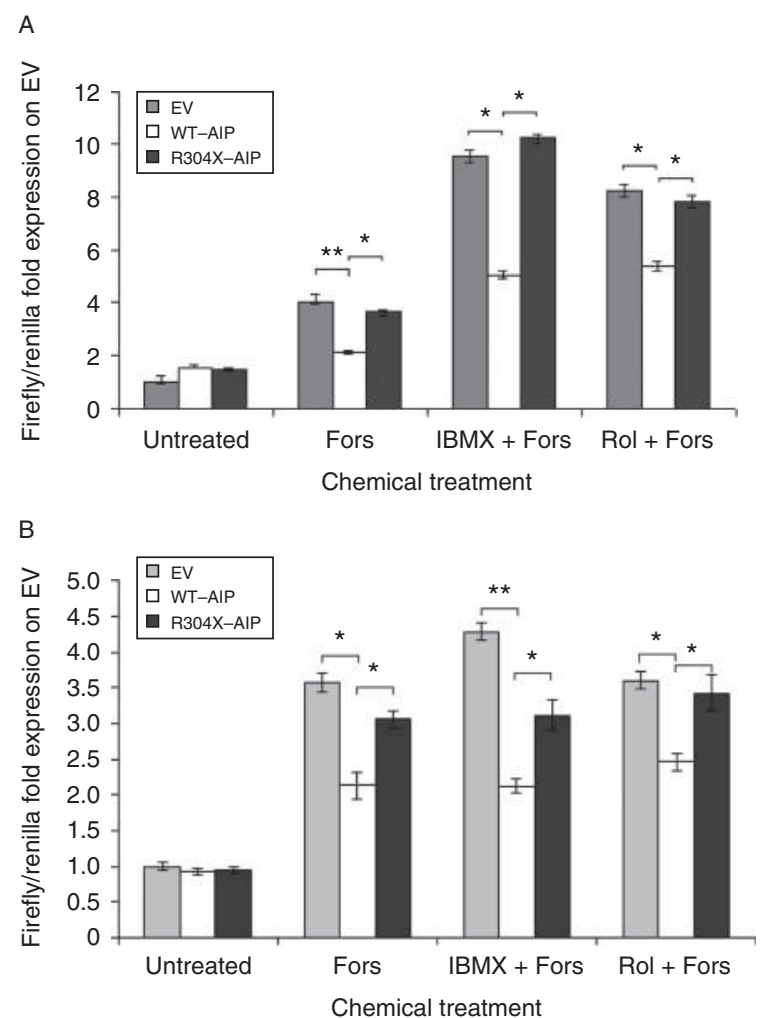

Figure 2

Wild-type (WT)-Aip reduced forskolin-induced CAMP-driven transcription using luciferase reporter activity in GH3 (A) and Hela cells (B) transfected with either empty vector (EV), WT or mutant R304X-AIP. Cells were left untreated, treated with $1 \mu \mathrm{M}$ forskolin only (Fors), $10 \mu \mathrm{M}$ IBMX followed by $1 \mu \mathrm{M}$ forskolin (IBMX+Fors) or $10 \mu \mathrm{M}$ rolipram followed by $1 \mu \mathrm{M}$ forskolin (Rol+Fors) before cell lysis. Bars represent means of three separate experiments with five replicates per experiment for the luciferase assays. Error bars indicate S.E.M. $(* P \leq 0.05, * * P \leq 0.005$; Fors, forskolin; Rol, rolipram).

Real-time PCR of a specific cAMP target gene, Id1, was also carried out in GH3 cells. Once again over-expression of WT or mutant Aip did not alter basal expression of Id1 gene significantly. Upon treatment with forskolin, Id1 mRNA expression increased threefold. However, WT-AIP-transfected cells showed a reduced elevation of Id1 mRNA when compared with those treated with either EV or R304X mutant AIP vector (Fig. 3). This effect was again retained when cells were pre-treated with IBMX. Thus, WT-Aip over-expressed in GH3 cells was able to reduce forskolin-induced cAMP target gene expression. Pre-treatment with rolipram resulted in the same observation, with WT-Aip over-expressing GH3 cells having a significantly lower Id 1 gene expression than differently treated cells.

Total cAMP was measured to assess whether overexpressed AIP reduced total cAMP or only its downstream

Published by Bioscientifica Ltd 


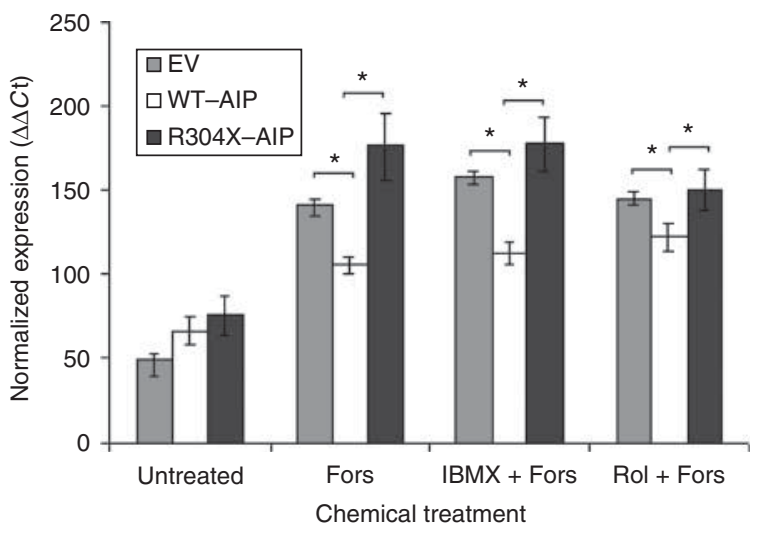

Figure 3

Wild-type (WT)-AIP reduced forskolin-induced CREB-driven Id 1 gene expression measured using real-time PCR. GH3 cells were transfected with empty vector (EV), WT or mutant R304X-AIP. Cells were either left untreated, treated with $1 \mu \mathrm{M}$ forskolin only (Fors), $10 \mu \mathrm{M}$ IBMX followed by $1 \mu \mathrm{M}$ forskolin (IBMX+Fors) or $10 \mu \mathrm{M}$ rolipram followed by $1 \mu \mathrm{M}$ forskolin (Rol+Fors) before RNA extraction. Bars represent three separate experiments with three replicates per experiment. Error bars indicate S.E.M. ( ${ }^{*} \leq \leq 0.05$; Fors, forskolin; Rol, rolipram).

effectors. Neither WT nor mutant Aip had any effect on basal cAMP levels. However, upon forskolin stimulation, the same trend was observed (Fig. 4A). WT-AIP reduced total cAMP when compared with both the EV and the R304X mutant. Addition of IBMX increased total cAMP significantly in cells transfected with WT-AIP. However, this was still significantly lower than that in EV and R304X-AIP-transfected GH3 cells treated with both IBMX and forskolin, although the difference was not as marked. Similar data resulted for the cells treated with rolipram before the addition of IBMX indicating that the effect of AIP over-expression on cAMP levels was not altered by the general or specific inhibition of phosphodiesterases.

As WT-AIP reduced total cAMP, it was decided to investigate whether this would influence GH secretion, which is directly influenced by cAMP levels. Basal GH secretion was not affected by the over-expression of the Aip gene. WT-AIP reduced forskolin-induced GH secretion compared with EV and R304X mutant AIP-transfected cells (Fig. 4B). Addition of IBMX caused a significant increase in GH secreted by WT-AIP over-expressing GH3 cells. However, the amount of GH secreted by these cells was still significantly lower than that secreted by GH3 cells transfected with the EV or R304X-AIP vector. Addition of rolipram also resulted in an overall increase in secreted GH, although WT-Aip over-expressing GH3 maintained a statistically lower GH secretion when compared with cells transfected with the EV or the R304X-AIP mutant.

\section{Knock down of endogenous Aip increases cAMP secondary messenger signalling}

Having established the effect of WT-Aip over-expression on cAMP signalling in GH3 cells, we wanted to assess whether the reverse behaviour would be observed by inhibiting the endogenous expression of the Aip gene. Figure $1 \mathrm{C}$ and D illustrates the success of silencing RNA knock down, which was specific to the rat Aip mRNA.

CRE-driven luciferase reporter activity and Id 1 target gene expression were measured after Aip knock down (Fig. 5A and B). As observed in these results, attenuation of the endogenous rat AIP protein resulted in a significant increase in cAMP-driven luciferase activity and Id1 gene expression even under basal conditions. GH3 cells transfected with rat Aip siRNA and chemically treated with $1 \mu \mathrm{M}$ forskolin also had significantly higher cAMP-driven
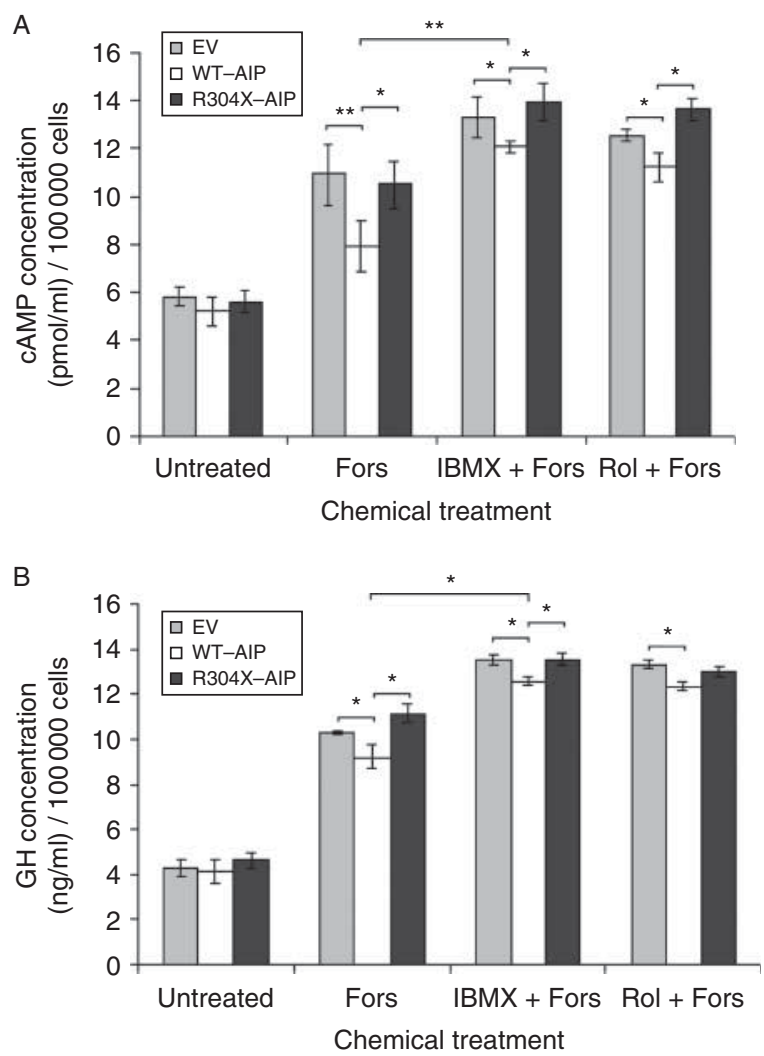

Figure 4

Wild-type (WT)-Aip over-expression reduced intracellular CAMP (A) and GH secretion (B) after stimulation by forskolin. $\mathrm{GH} 3$ cells were transfected with empty vector (EV), WT or mutant R304X-AIP and treated with $10 \mu \mathrm{M}$ IBMX/rolipram and/or $1 \mu \mathrm{M}$ forskolin $48 \mathrm{~h}$ after transfection. Medium was then collected for GH measurement and cells lysed for cAMP quantification. Bars represent means of three separate experiments with three replicates per experiment. Error bars indicate S.E.M. $\left({ }^{*} P \leq 0.05, * * P \leq 0.005\right.$; Fors, forskolin; Rol, rolipram).

Published by Bioscientifica Ltd. 

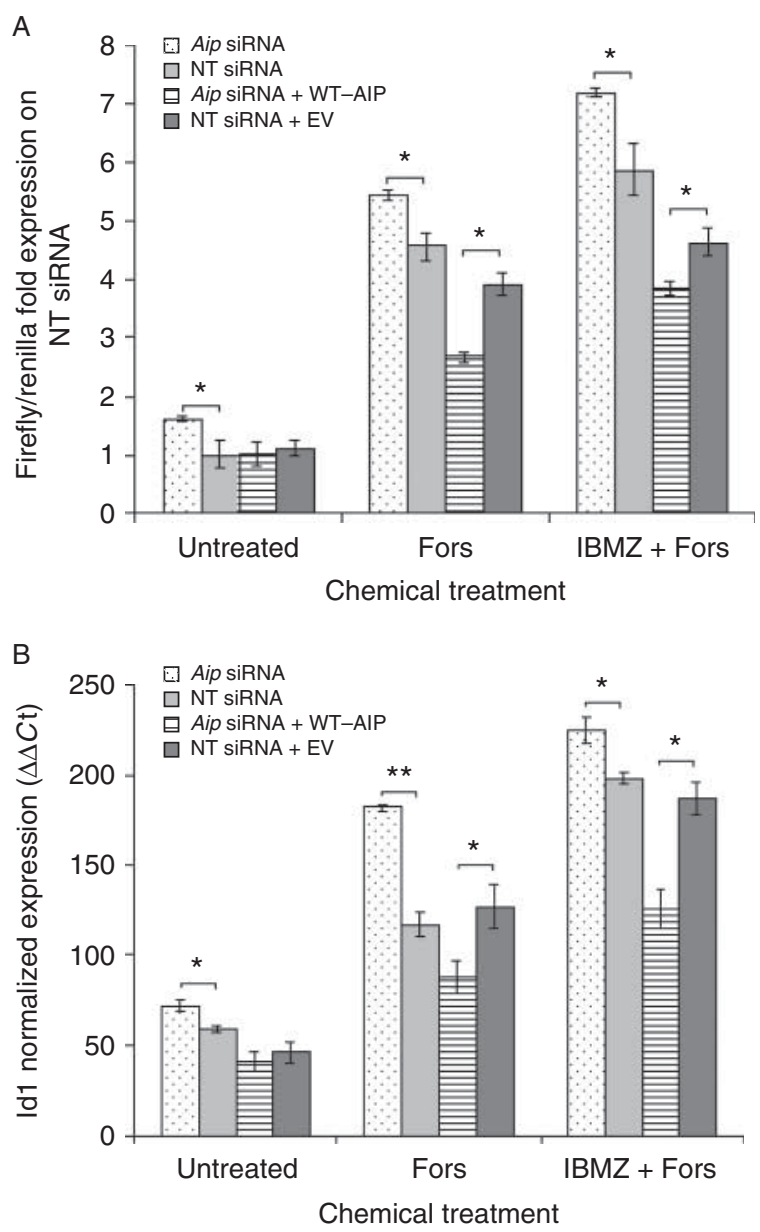

Figure 5

Knock down of endogenous rat Aip in GH3 cells increased cAMP-driven transcription of CRE-luciferase (A) and Id 1 cAMP target gene (B). GH3 cells were transfected with Aip siRNA or control non-targeting (NT) siRNA. After $48 \mathrm{~h}$, cells were left either untreated or treated with $1 \mu \mathrm{M}$ forskolin only or $10 \mu \mathrm{M}$ IBMX followed by $1 \mu \mathrm{M}$ forskolin before protein or RNA lysis. In the following setting, GH3 cells were co-transfected with Aip or NT siRNA and WT-AIP or empty vector (NT) and similarly treated. Bars represent means of three separate experiments with four replicates per experiment for the luciferase assays and three separate experiments with three replicates per experiment for the quantification of $I d 1$ expression. Error bars indicate S.E.M. $\left({ }^{*} P \leq 0.05, * * P \leq 0.005\right.$; Fors, forskolin; NT, non-targeting).

transcription than cells transfected with the non-targeting siRNA. In order to verify whether this effect could be reversed by the addition of WT-AIP over-expression, dual transfection with WT-AIP or EV plasmids was carried out. As observed for both the luciferase reporter assays and the $I d 1$ gene expression results, co-transfection with WT-AIP managed to mitigate the effect of endogenous Aip knock down under basal conditions and significantly reverse the effect upon treatment with forskolin only or IBMX followed by forskolin. Therefore, over-expression of human WT-Aip through expression vectors replaced the knocked down endogenous Aip and reversed its influence on cAMP downstream transcriptional activation.

An identical trend is observed when cAMP concentration was measured after rat Aip knock down (Fig. 6A). Inhibition of Aip expression results in increased cAMP concentration in untreated (not statistically significant), forskolin-treated and IBMX-pre-treated GH3 cells when compared with non-targeting siRNA-transfected cells. Once again, co-transfection with WT-Aip expression plasmid rescued the ablation of endogenous Aip knock down. Treatment with forskolin in Aip siRNA and WT-AIP plasmid co-transfected cells results in an overall significantly lower intracellular cAMP concentration when compared with cells co-transfected with non-targeting siRNA and an EV plasmid.

Interestingly, the trend observed in cAMP signalling and concentration under the influence of Aip knock down does not extend to GH secretion as observed in Fig. 6B. Transfection with Aip siRNA results in an overall reduction in GH secretion, even though statistical significance was not reached. GH3 cells transfected with rat Aip siRNA and treated with both IBMX and forskolin have a significantly lower GH concentration secreted in their medium when compared with cells with intact AIP expression. Co-transfection with WT-AIP plasmid was able to rescue this effect and only a slightly lower GH concentration was observed in cells over-expressing human AIP protein. However, treatment with forskolin in these cells expressing the plasmid AIP resulted in a significant reduction in GH secretion when compared with cells transfected with the non-targeting siRNA and EV. Similarly, pre-treatment with IBMX followed by forskolin resulted in a similar significant discrepancy.

\section{Discussion}

Germ-line Aip gene alterations predispose to pituitary adenoma formation. However, the mechanism of action remains unknown. This study aimed at characterising the putative protective function of AIP and analysing how genetic disruption may lead to increased susceptibility to pituitary cell neoplasia.

AIP, being a co-chaperone, interacts with a multitude of proteins, through which it can be speculated to play a protective role against pituitary tumorigenesis. Our study provides evidence that AIP influences the cAMP signalling pathway. PDE4A5, a cAMP-specific phosphodiesterase, has been reported to interact with AIP through the TPR region, resulting in a reduction in PDE4A5 catalytic

Published by Bioscientifica Ltd. 

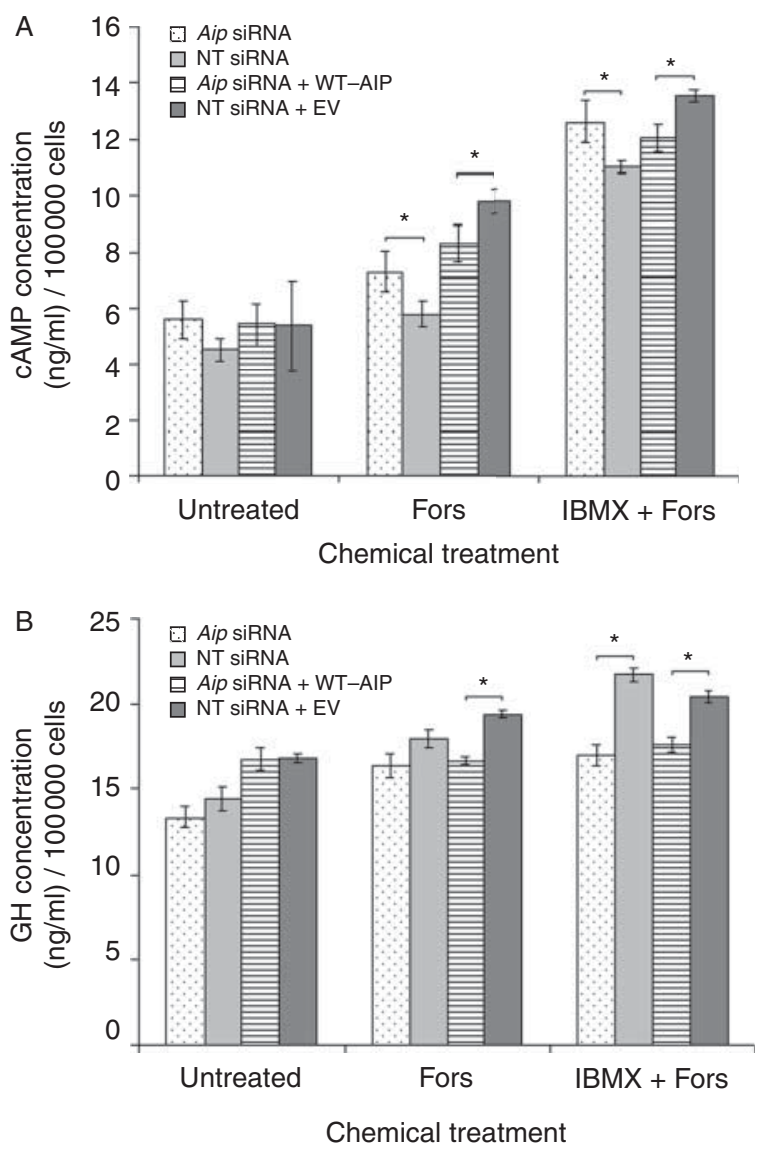

\section{Figure 6}

Knock down of endogenous rat Aip in GH3 cells increased the intracellular cAMP concentration (A) but it reduced GH secretion (B). GH3 cells were transfected with Aip siRNA or non-targeting (NT) siRNA. After $48 \mathrm{~h}$, cells were left either untreated or treated with $1 \mu \mathrm{M}$ forskolin only or $10 \mu \mathrm{M}$ IBMX followed by $1 \mu \mathrm{M}$ forskolin before collection of medium for $\mathrm{GH}$ assay or cell lysis for the CAMP assay. In the following setting, GH3 cells were co-transfected with Aip or NT siRNA and WT-AIP or empty vector (NT) and similarly treated. Bars represent means of three separate experiments with three replicates per experiment. Error bars indicate s.E.M. $\left({ }^{*} P \leq 0.05\right.$; Fors, forskolin; NT, non-targeting).

activity, decreased phosphorylation by PKA and increased sensitivity to the PDE4-specific inhibitor rolipram (Bolger et al. 2003). AIP also interacts with PDE2A, although this interaction did not affect PDE2A activity (de Oliviera et al. 2007). AIP protein was also found to interact with two specific $G \alpha_{13}$ and $G \alpha_{\mathrm{q}}$. Although these two proteins were initially thought to have no effect on cAMP signalling, one study reported that $\mathrm{G} \alpha_{13}$ activates an isoform of adenylate cyclase, type VII (AC7), which is partially responsible for cAMP production in a number of cells, including the pituitary (Jiang et al. 2007, 2008, Pronko et al. 2010). Given the lack of data with regard to interaction of the AIP protein with these four proteins, the effect of overexpression or knock down of AIP on cAMP signalling is a matter of speculation. The only well-characterised interaction reported was that between AIP and PDE4A5 in which AIP appeared to partially inhibit PDE4A5 activity. Therefore an increase in WT-AIP could be expected to increase cAMP levels by repressing PDE4A5. However, our data do not support this hypothesis.

Canonical cAMP signalling resulted in activation of the cAMP-response element binding protein (CREB) transcription factor (Yamamoto et al. 1988). Our study was designed to test the effect of AIP on cAMP effectors that bring about transcriptional changes. This was accomplished through the application of luciferase assays and quantitative PCR. Forskolin, used as a positive control, was shown in one study to increase AIP expression through PKA activation (Igreja et al. 2010). However, our study showed very low AIP expression in response to forskolin (Fig. 1A and B). The different forskolin concentrations used and the fact that a reporter assay with a putative AIP promoter sequence $(1.2 \mathrm{~kb}$ upstream of the AIP gene) was used in the aforementioned study could account for this discrepancy. Unfortunately, to date, there are no cell lines available that do not endogenously express AIP. Nonetheless, a significant increase in cAMP signalling was observed in forskolin stimulated as opposed to untreated cells in all experiments at the chosen concentration. It is possible that the relatively low cAMP activity in non-stimulated cells would preclude any discernible alterations. Another limitation could be the sensitivity of the assays used in this study.

Analysis of transcriptional activity clearly indicated the ability of WT-AIP to down-regulate forskolin-induced cAMP-driven transcription of luciferase in both GH3 and Hela cell lines or Id 1 expression in GH3 cells, a CREB target gene. Given the lack of human cell lines of the somatotroph/lactotroph lineage, Hela cells were used to verify the ability of WT-AIP to reduce cAMP signalling even in a human and a non-pituitary-derived cell line, therefore indicating that the behaviour of AIP is not limited to cells of the somatotroph/lactotroph lineage. The truncated R304X-AIP mutant, which lacks the C-terminal $\alpha-7$ region of the protein and was shown to be incapable of binding other proteins (Leontiou et al. 2008) lost such ability. This observation suggests that WT-AIP is able to suppress tumour growth and, in the case of this experimental setting, reduce cAMP signalling, by functionally interacting with other proteins, as would be expected of a co-chaperone. The similar behaviour of the R304X mutant to the EV indicates an almost complete loss of function in the setting of these experiments. The addition of IBMX increased cAMP-driven transcription.

Published by Bioscientifica Ltd 
However, GH3 cells over-expressing WT-AIP still had reduced cAMP activity when compared with the differently transfected cells. Rolipram, a selective PDE4 inhibitor, which decreases off-target effects when compared with IBMX, and selectively abolishes the AIP-PDE4A5 interaction, did not influence the ability of WT-Aip to reduce forskolin-induced cAMP-driven transcription in our study.

WT-Aip also reduced forskolin-induced cellular cAMP levels. The lack of effect on baseline cAMP levels and signalling suggests that the over-expressed WT-Aip achieves its effect by regulating cAMP production or limiting the cAMP production threshold. The inability of IBMX or rolipram to reverse the influence of WT-Aip on cAMP-driven transcription suggests that the AIP-PDE interaction is either not required or only partially responsible for the reduction in forskolin-activated cAMP signalling by WT-AIP. The reason for this discrepancy in the results could possibly be the fact that Aip interacts with a number of other proteins, besides the PDEs. Additionally, the study by Bolger et al. (2003) regarding the interaction between AIP and PDE4A5 was carried out in COS-7 cells, not GH3 or other neuroendocrine cell line, which could account for the conflicting results. The G-protein, $\mathrm{G} \alpha_{13}$, which is capable of stimulating cAMP production, provides a feasible alternative. However, little is known about the AIP-G $\alpha_{13}$ interaction, except that it causes a reduction in aryl hydrocarbon receptor (AHR) signalling (Jiang et al. 2008). cAMP also interacts with and activates the AHR (Oesch-Bartlomowicz et al. 2005), thereby offering another pathway through which the above mechanism could be explained. Additionally, AIP interacts with the thyroid hormone receptor $\beta 1$, altering its function (Froidevaux et al. 2006), and interestingly activated thyroid hormone receptors control GH transcription in pituitary cells (Spindler et al. 1989). Furthermore, the interaction of AIP with as yet unidentified proteins cannot be excluded.

GH secretion was also measured in order to determine whether the observed reduction in cAMP signalling would result in a relative reduction in GH secretion. Our study demonstrates that AIP reduces forskolin-induced, but not baseline, GH secretion, probably through a decrease in cAMP signalling, resulting in a proportional decrease in GH secretion (Fig. 4A and B). The AIP-G $\alpha_{13}$ interaction might therefore be responsible for this effect on cAMP production. GH3 cells over-expressing WT-AIP secreted significantly lower GH than GH3 cells over-expressing the mutant R304X Aip gene or the EV, once again confirming that the inhibition of phosphodiesterases does not impede the activity of AIP in down-regulating cAMP signalling and GH secretion.

Having observed the effect of WT and mutant Aip over-expression on cAMP signalling in GH3 cells, it was decided to verify whether knock down of endogenous rat AIP would lead to a reversal of the observed effects. Having accomplished successful knock down using a commercial silencing RNA composed of four siRNA sequences specific to the rat Aip gene, the same series of experiments were once again carried out to examine the status of cAMP signalling in GH3 cells so treated. As demonstrated in Fig. 4A and B, knock down of endogenous Aip gene gave results that were the opposite of those obtained in the over-expression studies. cAMP-driven transcription was significantly increased in both the luciferase reporter assay and the cAMP target gene analysis. Interestingly, this result was also observed under basal culture conditions. Treatment with forskolin or IBMX and forskolin confirmed the findings observed in the presence of reduced Aip expression.

In an attempt to verify whether the knock down of endogenous rat AIP could be rescued by the overexpression of human AIP, co-transfection experiments were carried out. As observed, cells co-transfected with rat Aip siRNA and human WT-AIP clearly show a reversal of the increase in cAMP signalling under siRNA treatment only in basal conditions. The addition of forskolin in the presence of over-expressed WT-AIP reverses the effect of the knock down and cells over-expressing WT-AIP have reduced cAMP-driven transcription similar to previous over-expression analysis, thereby nullifying the effect of the siRNA. These results indicate that human AIP protein can replace rat endogenous AIP and counteracts the loss of endogenous AIP in GH3 cells, causing a decrease in forskolin-stimulated cAMP signalling. Rat and human AIP proteins share a 95\% homology and are therefore likely to be able to completely or partially replace each other functionally. The ability of human WT-AIP overexpression to decrease cAMP signalling in the absence of endogenous Aip expression provides further proof of the functional impact of AIP on cAMP signalling and transcriptional potential in this in vitro context.

Further analysis of the effect of Aip knock down on cAMP levels in GH3 cells was also carried out. As observed in Fig. 6A, cells transfected with Aip siRNA showed a slight cAMP concentration increase under basal conditions, while statistical significance for this increase was only obtained when cells are treated with forskolin. Once again, co-transfection with human WT-AIP reverses this effect when cells are treated with forskolin only or IBMX

Published by Bioscientifica Ltd. 
followed by forskolin. This once again proves the ability of WT-AIP to influence not only cAMP signalling but possibly also its production.

Finally, an interesting and unexpected result was obtained with regard to GH from GH3 cells transfected with Aip siRNA. As observed in Fig. 6B, endogenous AIP knockdown resulted in a slight decrease in GH secretion under basal conditions. This result was obtained for all the cells whose endogenous Aip was knocked down by transfection with silencing RNA and statistical significance was obtained for cells treated with IBMX before forskolin addition. Such behaviour was contrary to our predictions that an increase in cAMP level would cause a relative increase in GH secretion. Although no specific explanation can be put forward to account for this behaviour, one possibility is the direct interaction between AIP and GH. Leontiou et al. (2008) reported that AIP and GH co-localise together in the secretory vesicles in somatotroph cells using gold immunostaining. Therefore, it is conceivable that AIP, acting as a co-chaperone, might have a direct role in regulating either GH stability or play a role in its secretion from somatotroph cells given its particular sub-cellular localisation. Although such an explanation requires further study, it would also account for the segregation of Aip mutations almost exclusively to somatotroph adenomas. This hypothesis would also provide another possible mechanism by which AIP plays a protective role in pituitary tumour formation.

Co-transfection of silencing RNA and WT-AIP overexpressing plasmids resulted in a further reduction of $\mathrm{GH}$ secretion when compared with siRNA-only transfected cells. In basal conditions, the presence of human AIP protein managed to almost restore normal GH secretion when compared with the non-targeting RNA and $\mathrm{EV}$ co-transfected cells. Stimulation with forskolin only or IBMX followed by forskolin once again resulted in a significant reduction in GH secretion. The reason for this is likely to be twofold. Primarily, the effect of endogenous Aip knock down leads to a slight reduction in GH secretion as observed in the siRNA-only transfected cells. Additionally, in co-transfected cells, the presence of over-expressed WT-AIP decreases further the release of GH, likely through the reduction in cAMP concentration of the GH3 cells as proven by previous results.

In conclusion, this study provides evidence for a novel mechanism through which AIP acts as a tumour suppressor by limiting cAMP signalling within pituitary cells. Given the abundant evidence for the mitogenic influence of cAMP signalling on pituitary cells, the ability of the AIP to reduce the intracellular cAMP and its downstream signalling may account for the tumour-suppressive role of AIP. Although baseline cAMP was not affected, forskolin-induced cAMP signalling was reduced in cells over-expressing WT but not mutant Aip. While this does not represent normal physiological conditions, somatotroph cells are known to undergo sudden increases in cAMP secondary to pulsatile secretion of hypothalamic GHRH, resulting in augmentation of pulsatile secretion of GH at certain times of the day (Melmed \& Conn 2005). AIP may therefore act by limiting the magnitude of these peaks and thus curb cAMP production and GH secretion during these peaks. Attenuation of AIP function by genetic mutation could cause magnification of such peaks, leading to increased cAMP signalling and GH production and secretion and thus increase the susceptibility towards pituitary tumour formation.

Ultimately, for such a mechanism to be proven beyond doubt, further experimentation, preferably in an in vivo model, would be required. Nonetheless, the evidence presented here by the over-expression and knock down of AIP respectively within this well-characterised cell line supports an interesting potential mechanism of action for this tumour suppressor protein. We have also shown that AIP may play a functional role in controlling $\mathrm{GH}$ secretion or production, whether by the reduction of cAMP levels or through a direct interaction with GH within the secretory granules of somatotroph cells remains to be seen. Our data indicates that the AIP-PDE interaction is not exclusively required for its tumour-suppressive behaviour to be observed, and given the nature of AIP as a co-chaperone, it is likely that it brings about its effect through a number of interactions, probably some of which remain as yet undiscovered.

\section{Declaration of interest}

The authors declare that there is no conflict of interest that could be perceived as prejudicing the impartiality of the research reported.

\section{Funding}

This research has been funded by the Malta Government Grant Scheme (Vote no. 88-019) and the University of Malta Research Fund Committee Allocation (Vote no. PHBRP07-01).

\section{Author contribution statement}

$\mathrm{R}$ Formosa was the main investigator and carried out all the functional analysis in relation to AIP and its mutations. A Xuereb-Anastasi is co-supervisor of the project and provided technical advice. J Vassallo is the main project coordinator and provided intellectual and technical supervision.

Published by Bioscientifica Ltd. 


\section{Acknowledgements}

The authors would like to thank Prof. M Korbonits, Dr Anthony Fenech and Prof. G Holz for the generous donation of materials used in this study. Special appreciation also goes to Dr Anthony Fenech, Dr Pierre SchembriWismayer, Dr Godfrey Grech and Prof. Gary Hunter for the use of facilities and precious assistance.

\section{References}

Beischlag TV, Morales JL, Hollingshead BD \& Perdew GH 2008 The aryl hydrocarbon receptor complex and the control of gene expression. Critical Reviews in Eukaryotic Gene Expression 18 207-250. (doi:10.1615/ CritRevEukarGeneExpr.v18.i3.20)

Bolger GB, Peden AH, Steele MR, MacKenzie C, McEwan DG, Wallace DA, Huston E, Baillie GS \& Houslay MD 2003 Attenuation of the activity of the cAMP-specific phosphodiesterase PDE4A5 by interaction with the immunophilin XAP2. Journal of Biological Chemistry 278 33351-33363. (doi:10.1074/jbc.M303269200)

Chepurny OG \& Holz GG 2007 A novel cyclic adenosine monophosphateresponsive luciferase reporter incorporating a nonpalindromic cyclic adenosine monophosphate response element provides optimal performance for use in $\mathrm{G}$ protein-coupled receptor drug discovery efforts. Journal of Biomolecular Screening 12 740-746. (doi:10.1177/ 1087057107301856)

Daly A, Tichomirowa M, Petrossians P, Heliovaara E, Jefrrain-Rea M, Barlier A, Naves LA, Ebeling T, Harhu A, Raappana A et al. 2010 Clinical characteristics and therapeutic responses in patients with germ-line AIP mutations and pituitary adenomas: an International Collaborative Study. Journal of Clinical Endocrinology and Metabolism 95 E373-E383. (doi:10.1210/jc.2009-2556)

Formosa R, Gruppetta M, Falzon S, Santillo G, DeGaetano J, Xuereb-Anastasi A \& Vassallo J 2012 Expression and clinical significance of Wnt players and survivin in pituitary tumours. Endocrine Pathology 23 123-131. (doi:10.1007/s12022-012-9197-8)

Froidevaux MS, Berg P, Seugnet I, Decherf S, Becker N, Sachs L, Bilesimo P, Nygard M, Pongratz I \& Demeneix BA 2006 The co-chaperone XAP2 is required for activation of hypothalamic thyrotropin-releasing hormone transcription in vivo. EMBO Reports 7 1035-1039. (doi:10.1038/sj.embor.7400778)

Horvath A \& Stratakis CA 2008 Clinical and molecular genetics of acromegaly: MEN1, Carney complex, McCune-Albright syndrome, familial acromegaly and genetic defects in sporadic tumours. Reviews in Endocrine \& Metabolic Disorders 9 1-11. (doi:10.1007/ s11154-007-9066-9)

Igreja S, Chahal HS, King P, Bolger GB, Srirangalingam U, Guasti L, Chapple JP, Trivellin G, Gueorguiev M, Guegan K et al. 2010 Characterization of aryl hydrocarbon receptor interacting protein (AIP) mutations in familial isolated pituitary adenoma families. Human Mutation 31 950-960. (doi:10.1002/humu.21292)

Jiang LI, Collins J, Davis R, Lin KM, DeCamp D, Roach T, Hsueh R, Rebres RA, Ross EM, Taussig R et al. 2007 Use of a cAMP BRET sensor to characterize a novel regulation of cAMP by the sphingosine 1-phosphate/G13 pathway. Journal of Biological Chemistry 282 10576-10584. (doi:10.1074/jbc.M609695200)

Jiang LI, Collins J, Davis R, Fraser ID \& Sternweis PC 2008 Regulation of cAMP responses by the G12/13 pathway converges on adenylate cyclase VII. Journal of Biological Chemistry 283 23429-23439. (doi:10.1074/ jbc.M803281200)

Kang BH \& Altieri DC 2006 Regulation of survivin stability by the aryl hydrocarbon receptor-interacting protein. Journal of Biological Chemistry 201 24721-24727. (doi:10.1074/jbc.M603175200)

Kazlauskas A, Poellinger L \& Pongratz I 2002 Two distinct regions of the immunophilin-like protein XAP2 regulate dioxin receptor function and interaction with hsp90. Journal of Biological Chemistry 277 11795-11801. (doi:10.1074/jbc.M200053200)

Lania A, Mantovani G \& Spada A 2001 G protein mutations in endocrine diseases. European Journal of Endocrinology 145 543-559. (doi:10.1530/ eje.0.1450543)

Lania AG, Mantovani G, Ferrero S, Pellegrini C, Bondioni S, Peverelli E, Braidotti P, Locatelli M, Zavanone ML, Ferrante E et al. 2004 Proliferation of transformed somatotroph cells related to low or absent expression of protein kinase a regulatory subunit $1 \mathrm{~A}$ protein. Cancer Research 64 9193-9198. (doi:10.1158/0008-5472. CAN-04-1847)

Leontiou CA, Gueorguiev M, van der Spuy J, Quinton R, Lolli F, Hassan S, Chahal HS, Igreja SC, Jordan S, Rowe J et al. 2008 The role of the AIP gene in familial and sporadic pituitary adenomas. Journal of Clinical Endocrinology and Metabolism 93 2390-2401. (doi:10.1210/jc.2007-2611)

Melmed S \& Conn PM 2005 Endocrinology: basic and clinical principles. 2nd edn. New York: Humana Press.

Nakata A, Urano D, Fujii-Kuriyama Y, Mizuno N, Tago K \& Itoh H 2009 G-protein signalling negatively regulates the stability of aryl hydrocarbon receptor. EMBO Reports 10 622-628. (doi:10.1038/ embor.2009.35)

Oesch-Bartlomowicz B, Huelster A, Wiss O, Antoniou-Lipfert P, Dietrcih C, Arand M, Weiss C, Bockamp E \& Oesch F 2005 Aryl hydrocarbon receptor activation by cAMP vs dioxin: divergent signaling pathways. PNAS 102 9218-9223. (doi:10.1073/pnas.0503488102)

Ohta Y, Nakagawa K, Imai Y, Katagiri T, Kioke T \& Takaoka K 2008 Cyclic cAMP enhances Smad-mediated BMP signaling through PKA-CREB pathway. Journal of Bone and Mineral Metabolism 26 478-484. (doi:10.1007/s00774-008-0850-8)

de Oliviera SK, Hoffmeister M, Gambaryan S, Muller-Esterl W, Guimaraes JA \& Smolenski AP 2007 Phosphodiesterase 2A forms a complex with the co-chaperone XAP2 and regulates nuclear translocation of the aryl hydrocarbon receptor. Journal of Biological Chemistry 282 13656-13663. (doi:10.1074/jbc.M610942200)

Pertuit M, Barlier A, Enjalbert A \& Gerard A 2009 Signalling pathway alterations in pituitary adenomas: involvement of Gs $\alpha$, cAMP and mitogen-activated protein kinases. Journal of Neuroendocrinology $\mathbf{2 1}$ 269-877.

Pronko SP, Saba LM, Hoffman PL \& Tabakoff B 2010 Type 7 adenylyl cyclase-mediated hypothalamic-pituitary-adrenal axis responsiveness: influence of ethanol and sex. Journal of Pharmacology and Experimental Therapeutics 334 44-52. (doi:10.1124/jpet.110.166793)

Ramirez JL, Castano JP, Torronteras R, Martinez-Fuentes AJ, Frawley LS, Garcia-Navarro S \& Gracia-Navarro F 1999 Growth hormone (GH) releasing factor differentially activates cyclic adenosine $3^{\prime}, 5^{\prime}$-monophosphate- and inositol phosphate-dependant pathways to stimulate GH release in two porcine somatotroph subpopulations. Endocrinology 140 1752-1759. (doi:10.1210/en.140.4.1752)

San-Marina S, Han Y, Suarez Saiz F, Trus MR \& Minden MD 2008 Lyl1 interacts with CREB1 and alters expression of CREB1 target genes. Biochimica et Biophysica Acta 1783 503-517. (doi:10.1016/j.bbamcr. 2007.11.015)

Spindler SR, Crew MD \& Nyborg JK 1989 Thyroid hormone transcriptional regulatory region of the growth hormone gene. Endocrine Research $\mathbf{1 5}$ 475-493. (doi:10.3109/07435808909036349)

Suzuki S, Yamamoto I \& Arita J 1999 Mitogen-activated protein kinasedependant stimulation of proliferation of rat lactotrophs in culture by $3^{\prime}, 5^{\prime}$-cyclic adenosine monophosphate. Endocrinology 140 2850-2858. (doi:10.1210/en.140.6.2850)

Vierimaa O, Goergitsi M, Lehtonen R, Vahteristo P, Kokko A, Raitila A, Tuppurainen K, Ebeling TML, Salmela PI, Paschke R et al. 2006 Pituitary adenoma predisposition caused by germline mutations in the AIP gene. Science 312 1128-1230. (doi:10.1126/ science.1126100) 
Yamamoto KK, Gonzalez GA, Biggs WH \& Montminy MR 1988 Phosphorylation-induced binding and transcriptional efficacy of nuclear factor CREB. Nature 334 494-498. (doi:10.1038/334494a0)

Yang J, Li X, Al-Lamki RS, Southwood M, Zhao J, Lever AM, Grimminger F, Schermuly RT \& Morrell NW 2010 Smad-dependent and smadindependent induction of id1 by prostacyclin analogues inhibits proliferation of pulmonary artery smooth muscle cells in vivo.
Circulation Research 107 252-262. (doi:10.1161/CIRCRESAHA.109. 209940)

Zhang X, Odom DT, Koo SH, Conkright MD, Canettieri G, Best J, Chen H, Jenner R, Herbolsheimer E, Jacobsen E et al. 2005 Genome-wise analysis of cAMP-response element binding protein occupancy, phosphorylation, and target gene activation in human tissues. PNAS $\mathbf{1 0 2}$ 4459-4464. (doi:10.1073/pnas.0501076102)

Received in final form 19 April 2013

Accepted 20 May 2013

Made available online as an Accepted Preprint

23 May 2013
Published by Bioscientifica Ltd. 